\title{
The Hardware-In-The-Loop simulation system of the diesel generator set based on the NARMAX model
}

\author{
Shanshan Ai \\ School of Aerospace Engineering, Beijing Institute of \\ Technology \\ Beijing, china \\ allie.999@163.com \\ Shuhua Zheng \\ School of Automation, Beijing Institute of Technology \\ Beijing, china \\ zhengshuhua@bit.edu.cn
}

\author{
Bo Mo \\ School of Aerospace Engineering, Beijing Institute of \\ Technology \\ Beijing, china \\ mobo@bit.edu.cn \\ Mingxin $\mathrm{Yu}$ \\ School of Automation, Beijing Institute of Technology \\ Beijing, china \\ mingxinbit@yahoo.cn
}

\begin{abstract}
The control of the speed of the diesel engine is the key to guarantee the AC output quality of the DGS (Diesel Generator Set). Because of the heavy noise and pollution of the physical DGS, it is a good way to build the mathematical model for research on the control of the diesel engine output speed. But the theoretical models often were not accurate and their control algorithms had poor usability due to nonlinearity and soft parameters of the diesel engine. To solve these problems, a Hardware-in-the-Loop (HIL) simulation system of the DGS has been built in this paper. In the HIL system, the power unit (includes diesel engine, generator and load tank) is replaced by the mathematic model, and the other devices are same as ones in the physical DGS. In order to ensure the accuracy of the model, this paper proposes modeling method for the power unit of the DGS. Thanks to the NARMAX (Nonlinear AutoRegressive Moving Average with eXogenous inputs) model's good ability to describe the nonlinear system, the measurement data of the power unit are used to training NARMAX model and a high accuracy model of the power unit has been obtained. The experiments on the HIL system show the model can reproduce the static and dynamic characteristic of the physical DGS power unit to meet the research needs of the electronic governor.
\end{abstract}

Keywords- Diesel generator set; Electronic governor; LabVIE W; NARMAX model; Hardware-in-the-loop.

\section{INTRODUCTION}

Diesel generator sets (DGSs) are found in use across the world providing primary power or as backup supplies during mains outages, or even providing network support functions. Thanks to their flexibility, the DGSs are an essential method of generation in any power system. But the experiments of the DGS have the shortcomings of heavy noise and pollution. These problems can be solved by the Hardware-in-the-Loop (HIL) simulation system, in which the diesel engine model replaces the physical diesel engine. On the other hand, the electronic governor is a critical component in the DGS, when the parameters of the governor cannot meet the diesel engine characteristics, the DGS can't work normally. So the model of the diesel engine also has the great significance to debug the electronic governor. The HIL can speed up the development of electronic governor and save the energy in process of the DGS experiments.

Many researchers proposed models of the diesel engines for the research of their control algorithm. The models of diesel engines have two categories.

Firstly, the models are achieved by taking into account physical equations which govern the process, so a deep knowledge of the diesel engine is necessary [1-4]. Because the diesel engine is both a thermodynamic device and a kinematical device, so many dynamics within the running process of the diesel engine, such as intake and exhaust dynamic, fueling dynamics, combustion and heat release, heat transfer and power production. Therefore, the model building needs to a large number of parameters. However, it is very difficult and hard to achieve those parameters by calculation or identification. Those models are usually too complex to be used for the controller development.

Secondly, the parametric and non-parametric models are proposed for simplifying the models. The parametric models are derived by physical principle and relatively few physical parameters. The models also called grey-box modeling. The non-parametric models are built by a priori chosen structure and the input-output behaviors of the diesel engine [5-9]. This paper chooses non-parametrical modeling method.

The organization of this article is as follows. The whole structure of the hardware-in-the-loop system is described in section II. In section III, the NARMAX model structure is presented. The section IV depicts how to deal with the experiment data about the model. In section $\mathrm{V}$, the NARMAX is derived. The section VI is about the HIL simulation consequence through the regulation of the controller. Lastly, we give the conclusions and significance of our results in section VII.

\section{THE STRUCTURE OF THE HIL SYSTEM}

The DGS HIL system consists mainly of the electronic speed governor, the actuator, the speed pick-up sensors, the signal conditioning modules and the Ac servo motor system. 
The electronic speed governor generates a PWM output according to the feedback signal from the pick-up sensor to drive the actuator. In the HIL system, the DGS is substituted by its model and simulated in real time. In the system, the power unit, which is assembled by the diesel engine and the driveline, is seen as a system with double input and single output. The inputs are rack displacement and electrical load, the single output is the speed of the diesel engine. The model outputs the speed value for setting the rotational speed of the $\mathrm{AC}$ servo motor. The output of the pick-up sensor, which is centered rightly on the gearwheel driven by the AC servo motor, is as the real input value to the electronic speed governor. The output from the displacement sensor, which is rigidly linked to the actuator, is seen as the rack displacement of the diesel engine. The structure of system is shown as Fig. 1.

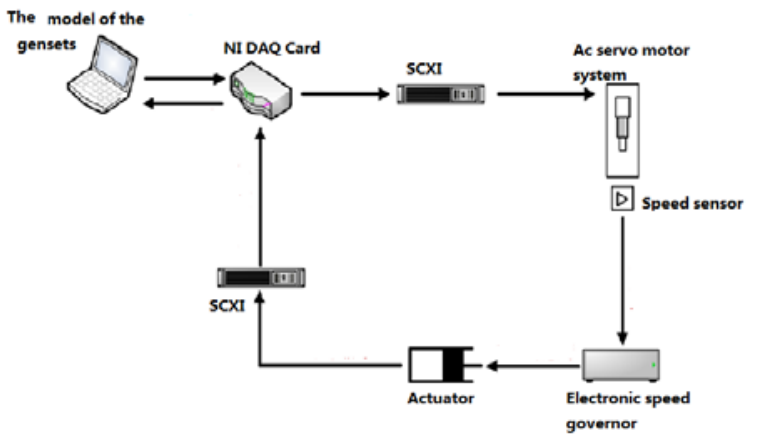

Figure 1. The structure of the system

\section{THE NARMAX MODEL}

The NARMAX approach is a good means of describing the input-output relationship of a nonlinear system. The model represents the extension of the well-known ARMAX model [10] to the nonlinear case, and it can be represented by (1) as follow.

$$
\begin{gathered}
y(k)=F^{\ell}\left[y(k-1), \cdots, y\left(k-n_{y}\right), u\left(k-\tau_{d}\right), \cdots\right. \\
u\left(k-\tau_{d}-n_{u}\right), e(k), e(k-1) \\
\left.e\left(k-n_{e}\right)\right]
\end{gathered}
$$

Where $\mathrm{n}_{\mathrm{y}}, \mathrm{n}_{\mathrm{u}}$ and $\mathrm{n}_{\mathrm{e}}$ are the maximum lags considered for the output $\mathrm{y}(\mathrm{k})$, input $\mathrm{u}(\mathrm{k})$, and noise $\mathrm{e}(\mathrm{k})$ respectively, $\tau_{\mathrm{d}}$ is the delay and $\mathrm{F}^{\ell}$ is a nonlinear mapping, $\mathrm{F}$ is a nonlinear function and $\mathrm{L}$ is the nonlinearity degree. Variables $\mathrm{u}(\mathrm{k}), \mathrm{y}(\mathrm{k})$ designate the system input and output, respectively, at the sampling instants, $\mathrm{e}(\mathrm{k})$ accounts for uncertainties, possible noise, non-modeled dynamics, and so on.

A polynomial expansion of equation (1) is

$$
\begin{aligned}
y(\mathrm{k}) & =\theta_{0}+\sum_{i_{1}=1}^{n} \theta_{i_{1}} s_{i_{3}}(\mathrm{k}) \\
& +\sum_{i_{1}=1}^{n} \sum_{i_{2}=i_{1}}^{n} \theta_{i_{1} i_{2}} S_{i_{1}}(\mathrm{k}) s_{i_{2}}(\mathrm{k}) \\
& +\sum_{i_{1}=1}^{n} \cdots \sum_{i_{l}=i_{l-1}}^{n} \theta_{i_{1} \cdots i_{l}} S_{i_{1}}(\mathrm{k}) \cdots S_{i_{l}}(\mathrm{k})
\end{aligned}
$$

Where the degree of nonlinearity $\mathrm{L}$ is the maximum order of the monomials in the model, and

$$
\begin{aligned}
& \mathrm{s}_{1}(\mathrm{k})=\mathrm{y}(\mathrm{k}-1), \mathrm{s}_{2}(\mathrm{k})=\mathrm{y}(\mathrm{k}-2), \cdots, \\
& \mathrm{s}_{n_{y}+1}(\mathrm{k})=\mathrm{u}(\mathrm{k}-\mathrm{d}), \cdots, \mathrm{s}_{n_{y}+n_{u}+1}(\mathrm{k})=\mathrm{e}(\mathrm{k}-1), \\
& \cdots, \mathrm{s}_{n}(\mathrm{k})=\mathrm{e}\left(\mathrm{k}-\mathrm{n}_{e}\right)
\end{aligned}
$$

In this paper, the system is a double input and single output system, we regard $y(k)$ as the output and $\mathrm{u}_{\mathrm{d}}(\mathrm{k}), \mathrm{u}_{\mathrm{t}}(\mathrm{k}) \mathrm{u}_{\mathrm{d}}(\mathrm{k}) \quad$ as inputs, as well as ignore the noise $\mathrm{e}(\mathrm{k})$. The maximum time lags in each variable are $n_{y}, n_{u 1}$ and $n_{u 2}$. In this brief, the model structure of the NARMAX polynomials is automatically chosen using the error reduction ratio (ERR) criterion [11], [12]. In the context the parameters of such models are obtained by the orthogonal least squares algorithm.

\section{EXPERIMENT DATA}

The experiments were performed in the physical DGS with $30 \mathrm{~kW}$ rated power. The data are acquired from the pickup sensor and the displacement sensor of the actuator on the running of the DGS. The load bank is respectively programmed to no-load, $25 \%, 50 \%, 75 \%$, and $100 \%$ load quantity of rated power $30 \mathrm{~kW}$. The experiment data are presented in Fig.2. These data will be used to estimate the model parameters.

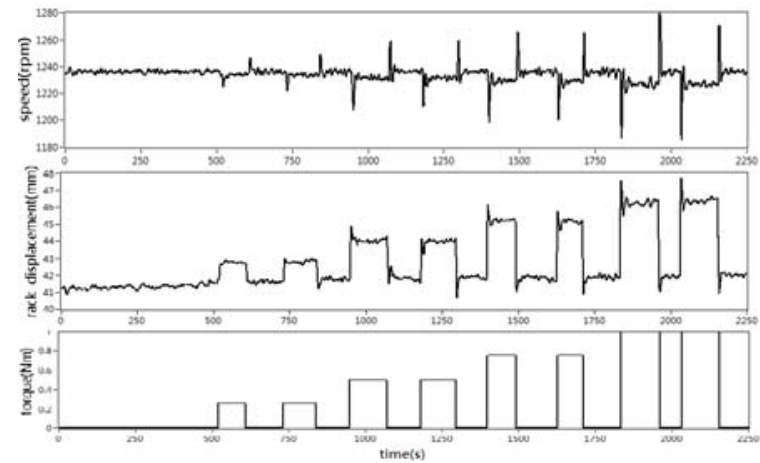

Figure 2. Training data of the model

V. THE MODEL OF THE DIESEL

In this part, the model of the power unit is constructed via the NARMAX methods. 


\section{A. The NARMAX structure of the diesel model}

The NARMAX model can well describe nonlinear systems in terms of linear-in-parameters difference equations relating the current output combinations of inputs and past outputs. The kind of model is suitable and capable to describe a wide variety of nonlinear systems. The structure selection is a key problem in system identification. The identification of the NARMAX model is complex through the classic least square method. The orthogonal least squares algorithm presented by Billings et al is used to perform both model structure detection and parameter estimation. This method process a linear-in-the-parameters polynomial by assessing the significance of all the possible monomials in the polynomial by the ERR. A monomial with a higher ERR indicates that it has a higher contribution to the system. The algorithm then implements a least squares method to estimate the coefficients of significant monomials. The detailed information about it can be found in [11].

In this paper, the NARMAX model is built using the degree of nonlinearity $\mathrm{L}=2$, the maximum lags used were $n_{y}=n_{u 1}=n_{u 2}=5$ , and the dimension $n=n_{y}+n_{u 1}+n_{u 2}$. Using the least square method, we can get the model of the diesel power unit as shown follow:

$$
\begin{aligned}
y(\mathrm{k})= & \theta_{2} \mathrm{y}(\mathrm{k}-1)-\theta_{3} \mathrm{y}(\mathrm{k}-2)-\theta_{4} \mathrm{y}(\mathrm{k}-3) \\
+ & \theta_{5} \mathrm{y}(\mathrm{k}-4)+\theta_{6} \mathrm{y}(\mathrm{k}-5)-\theta_{7} \mathrm{u}_{\mathrm{d}}(\mathrm{k}-1) \\
- & \theta_{8} \mathrm{u}_{\mathrm{d}}(\mathrm{k}-2)-\theta_{9} \mathrm{u}_{\mathrm{d}}(\mathrm{k}-3)+\theta_{10} \mathrm{u}_{\mathrm{d}}(\mathrm{k}-4) \\
+ & \theta_{11} \mathrm{u}_{\mathrm{d}}(\mathrm{k}-5)-\theta_{12} \mathrm{u}_{\mathrm{t}}(\mathrm{k}-1)+\theta_{13} \mathrm{u}_{\mathrm{t}}(\mathrm{k}-2) \\
- & \theta_{14} \mathrm{u}_{\mathrm{t}}(\mathrm{k}-3)+\theta_{15} \mathrm{u}_{\mathrm{t}}(\mathrm{k}-4)-\theta_{16} \mathrm{u}_{\mathrm{t}}(\mathrm{k}-5) \\
& +\theta_{87} \mathrm{u}_{\mathrm{d}}(\mathrm{k}-1) \mathrm{u}_{\mathrm{t}}(\mathrm{k}-1) \\
& -\theta_{97} \mathrm{u}_{\mathrm{d}}(\mathrm{k}-2) \mathrm{u}_{\mathrm{t}}(\mathrm{k}-2) \\
& +\theta_{106} \mathrm{u}_{\mathrm{d}}(\mathrm{k}-3) \mathrm{u}_{\mathrm{t}}(\mathrm{k}-3) \\
& -\theta_{114} \mathrm{u}_{\mathrm{d}}(\mathrm{k}-4) \mathrm{u}_{\mathrm{t}}(\mathrm{k}-4) \\
& +\theta_{121} \mathrm{u}_{\mathrm{d}}(\mathrm{k}-5) \mathrm{u}_{\mathrm{t}}(\mathrm{k}-5)
\end{aligned}
$$

In Fig. 3 and Fig. 4 are the detail model fittings at $75 \%$, $100 \%$ sudden load and sudden unload respectively.

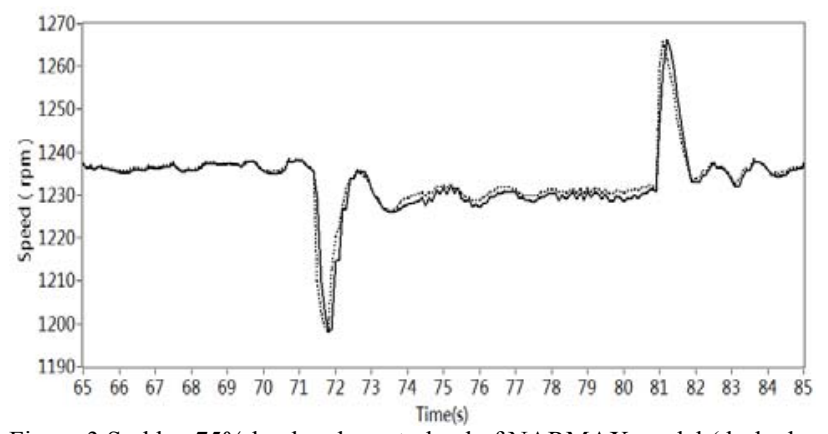

Figure. 3 Sudden $75 \%$ load and empty load of NARMAX model (dashed line) and the corresponding measured data (solid line)

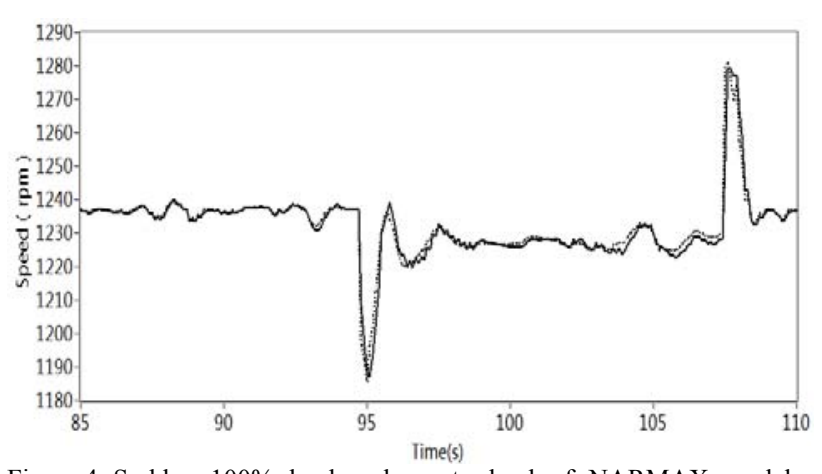

Figure.4 Sudden $100 \%$ load and empty load of NARMAX model (dashed line) and the corresponding measured data (solid line)

\section{B. The output accuracy of the DGS power unit}

It is not obvious that how to quantify the analysis of the model simulated data with the measurements. In order to solve this problem, the equation (5) is used to evaluate the accuracy of model output.

$$
\% V A F=\left[1-\frac{\frac{1}{N} \sum_{n=1}^{N}(y(n)-\hat{y}(n))^{2}}{\frac{1}{N} \sum_{n=1}^{N}(y(n))^{2}}\right] \times 100 \%
$$

Where $\mathrm{N}$ is the record length, $y(\mathrm{k})$ is the measured data, $\hat{y}(\mathrm{k})$ is the model output. The simulation output $\hat{y}(\mathrm{k})$ of the NARMAX description model is compared with the output of the measured data $y(\mathrm{k})$ by computing the percent variance for $\% \mathrm{VAF}$.

Fig.3 and Fig.4 show the simulation input (solid line) and predicted output (dashed line) of the NARMAX description model. With over 99\%VAF the NARMAX output matched that of the simulation with negligible error. So we will use the NARMAX model in the HIL simulation.

\section{THE SIMULATION ANALYSIS}

\section{A. Simulation setup}

The identification algorithm presented in the previous section is applied to the HIL simulation system with The Lab VIEW environment.

\section{B. The simulation based on controller}

The electronic governor as the controller in the HIL simulation system is to guarantee stability operation of the whole system.

Satisfactory closed-loop performance requires the settling time of less than 3 seconds, the steady-state error of less than $0.5 \%$, and the maximal speed drop of less than $5 \%$ on shut of a $100 \%$ load from empty load. A nonlinear closed-loop simulation and a HIL controller implementation are used to validate whether the desired closed-loop characteristics are met. 
The power unit model uses the previous NARMAX model. The actuator and the governor in the HIL simulation system are the ADB225 and ESD-5100 respectively, made in GAC, USA. The experiments investigated through the variable load, such as $100 \%, 75 \%, 50 \%, 25 \%$ and $0 \%$ of the full load. The governor has the good control effects (Fig.5) when sudden load $(100 \%, 75 \%, 50 \%, 25 \%)$ and empty load, and the speed can recover to the setting value with 2.5 time.

Fig. 6 shows the double input and single output curves of the HIL system in the 30 seconds, which included in 10 second and in 20 second sudden $100 \%$ load and sudden $100 \%$ unload respectively.

In Fig.6, the load is exerted on the DGS at 10 second, and the speed fall down to 1150rpm suddenly, then the speed rises and steadies in the setting speed through electronic governor control, the regulating time is $2.12 \mathrm{~s}$. At 20 second, the speed rises to $1260 \mathrm{rpm}$ suddenly when the load was discharged, the speed revived to the setting value after $2.14 \mathrm{~s}$. The experiments show the steady-state regulation ratio is $0.05 \%$, the transient regulation ratio of sudden unload is $4.47 \%$. So the model and the controller can meet the requirement.

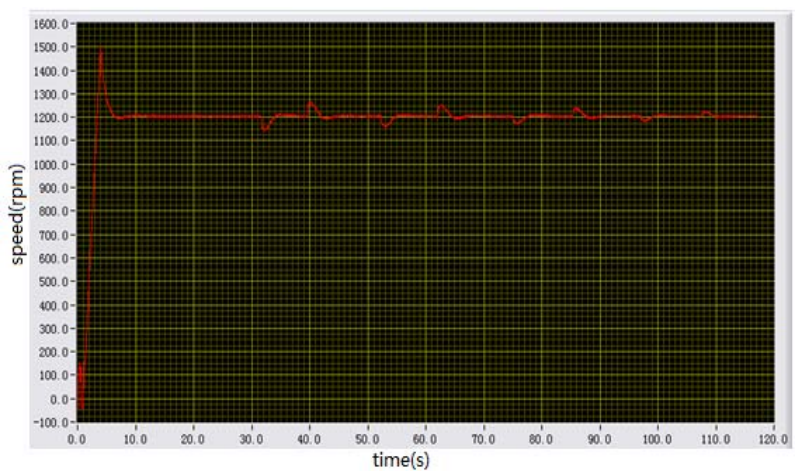

Figure.5. The speed with the variable load $(100 \%, 75 \%, 50 \%, 25 \%$ sudden load and sudden unload)

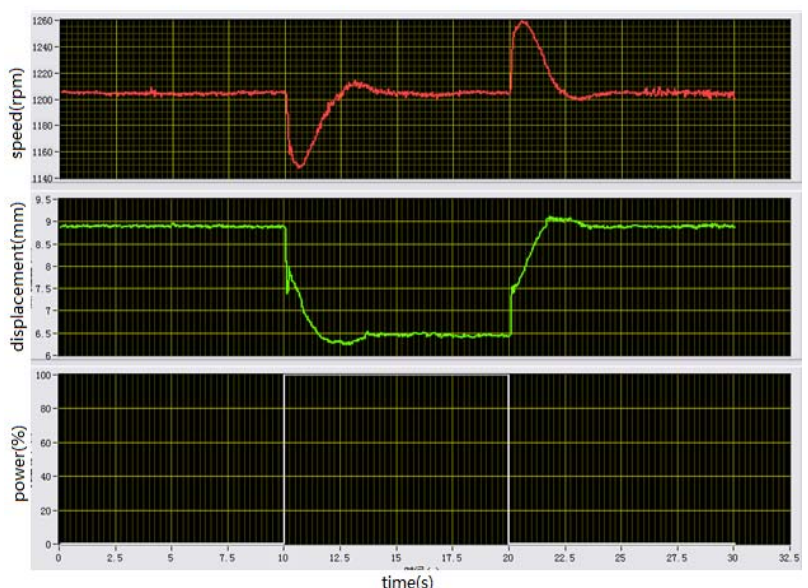

Figure. 6 The curves of speed, displacement and power in the hardwarein-the-loop system simulation based on the NARMAX model

\section{THE CONCLUSION}

The measurement data of the rack displacement and speed of the diesel engine and the electrical load from the physical DGS are used to train the NARMAX model for building the power unit model. The NARMAX model has a $99 \%$ VAF by the model accuracy assessment criteria. So the NARMAX models can recurrence the static and dynamic characteristic of the power unit in the HIL system. The experiments on the HIL system show that the HIL system can provide a green and flexible platform for the electric governor researches.

\section{REFERENCES}

[1] A. Palianos, A. Achir, R. Stobart, N. Langlois, and H. Chafouk, "Dynamic feedback linearization based control synthesis of the turbocharged diesel engine," American Control Conference, vol.10, pp. 4407-4412, 2007.

[2] S. Chen and J. Moskwa, "Application of Nonlinear Sliding-mode Observers for Cylinder Pressure Reconstruction," Control Engineering Practice, vol.5, pp.1115-1121, August. 1997.

[3] J. Jensen, A. Kristensen, S. Sorenson, N. Houbak, and E. Hendricks, "Mean value modeling of a small turbocharged Diesel engine," SAE paper, no. 911843, 1991.

[4] A. Rachid, A. Lizid, and J. Champussin, "Nonlinear modeling of a turbocharged diesel engine," Proc. IEEE Conf. Control Applications, Piscataway, vol.1, pp.133-136, 1994.

[5] K. Cheong, P. Li, and X. Jicheng, "Control oriented modeling and system identification of a diesel generator set (genset)," American Control Conference, pp. 950-955, 2010.

[6] H. Kuraoka, N. Ohka, M. Ohba, S. Hosoe, and F. Zhang, "Application of H-infinity design to automotive fuel control," IEEE Control System Magazine, vol. 10, no. 3, pp. 102-106, 1990.

[7] J. Jiang, "Optimal gain scheduling controller for a diesel engine," IEEE Control System Magazine, vol. 14, no. 4, pp. 42-48, 1994.

[8] G. Zito and I. Landau, "Narmax model identification of a Variable Geometry Turbocharged diesel engine," American Control Conference, pp. 1021-1027, JUNE. 2008.

[9] A. Rachid, A. Liazid, and J. Champoussin, "Nonlinear modeling of a turbocharged diesel engine," Control Applications, Proceedings of the Third IEEE Conference on, vol. 1, pp. 133-136, 1994.

[10] S.A.Billings, S.Chen, Representation of non-linear systems: the NARMAX model, International Journal of Control 49(3) (1989) 1013-1032

[11] S.A.Billings, S.Chen, and M.J.Korenberg, "Identification of MIMO nonlinear systems using a forward-regression orthogonal estimator," Int.J.Control, vol.49, no.6, pp.2157-2189,1989

[12] S.Chen, S.A.Billings, and W.Luo, "orthogonal least squares methods and their application to nonlinear system identification", Int.J.Control, vol.50, no.5, pp.1973-1896,1989. 